\title{
Role of Hormone Replacement Therapy in Young Women Suffering from Menopausal Symptoms of Cancer -A Concise Review
}

\section{Lokanatha, K. Govind Babu, Gita R. Bhat*}

Department of Medical Oncology, Kidwai Memorial Institute of Oncology, Bangalore, India Email: "Livewire841@gmail.com

Received 12 November 2015; accepted 28 November 2015; published 3 December 2015

Copyright (C) 2015 by authors and OALib.

This work is licensed under the Creative Commons Attribution International License (CC BY). http://creativecommons.org/licenses/by/4.0/

\section{(c) (i) Open Access}

\section{Abstract}

Cancer survivors are faced with several survivorship issues. In addition, young women may have to face the challenges brought on by premature menopause. The issue of Hormone Replacement Therapy (HRT) in these young ladies has always been a matter of debate. This is based on the theory that HRT could stimulate the growth of residual cancer cells and lead to recurrence. This article reviews the current recommendations for the use of HRT in various cancers. We also discuss the newer hormonal replacement therapies.

\section{Keywords}

Premature Menopause, Cancer Survivors, Hormone Replacement Therapy

Subject Areas: Gynecology \& Obstetrics, Oncology, Women's Health

\section{Introduction}

Cancer survivors are plagued by survivorship issues such as cardiac problems, second malignant neoplasms, weight gain, cognitive impairment, fatigue, psychological distress and depression [1]. Additional survivorship issues faced by premenopausal women include amenorrhea, infertility, early menopause, sexual dysfunction, osteopenia and osteoporotic fractures [2]. This article reviews the current recommendations for the use of HRT in various cancers. The newer hormonal therapies have also been discussed.

\footnotetext{
${ }^{*}$ Corresponding author.
}

How to cite this paper: Lokanatha, D., Govind Babu, K. and Bhat, G.R. (2015) Role of Hormone Replacement Therapy in Young Women Suffering from Menopausal Symptoms of Cancer-A Concise Review. Open Access Library Journal, 2: e2069. http://dx.doi.org/10.4236/oalib.1102069 


\section{What Are the Challenges Faced by Young Women with Menopausal Symptoms of Cancer and What Is Their Prevalence?}

In this setting, menopausal symptoms are similar to those experienced at natural menopause. However, they are more abrupt and persistent [3]. They include:

1) Hot flashes: A hot flash is defined as a subjective sensation of heat along with the objective signs of cutaneous vasodilatation and a subsequent drop in core temperature. It may be accompanied by sweating, palpitations, anxiety, night sweats, irritability and panic. It may last a few seconds to several minutes and may vary in severity from mild to intolerable. It varies in frequency from symptoms occurring daily to several times a month [4] [5].

2) Symptoms of genitourinary atrophy: Vaginal dryness and resultant dyspareunia lead to decreased desire and arousal.

3) Sexual dysfunction: Sexual dysfunction results in depression, anxiety and vice versa. It has a negative impact on the quality of life. It is often not discussed with survivors, thereby adding to their distress [1].

4) Emotional upset: Weight gain and unhappiness with body appearance could lead to psychological distress.

5) Musculoskeletal symptoms: These women have loss of bone mineral density. Osteoporosis and arthralgia may be worsened by aromatase inhibitors [3].

In 1997, the University of California at Los Angeles initiated the Cancer and Menopause Study (CAMS). They evaluated the quality of life and health outcomes of young female survivors of breast cancer. The study focused on the reproductive and late treatment-related side effects. The prevalence of hot flashes ranged from $20 \%$ in those aged 25 to 34 years to $65 \%$ in those aged 45 to 51 years. Similarly vaginal dryness and dyspareunia were aged related, ranging from $20 \%$ in those aged 25 to 34 years to 55\% in those aged 45 to 51 years [6].

Patients may have genetic predispositions to severe symptoms which are a focus of current research. Not only do these symptoms compromise the quality of life, they may affect adherence to endocrine therapies. Thus those whose side effects are poorly managed maybe at an increased risk of recurrence [7].

\section{Definition of Hormone Replacement Therapy (HRT)}

HRT is defined as the administration of natural or synthetic female hormones to compensate the diminution or deprivation of natural hormones. Estrogen alone (ERT) is used in a woman whose uterus has been removed. In a woman with an intact uterus, Estrogen is combined with a Progestin (EPT), so as to prevent endometrial proliferation [8].

\section{Why Do We Need to Add a Progestin?}

Estrogen leads to upregulation of estrogen/progesterone receptors and cellular mitosis in endometrial glandular epithelium. This leads to endometrial proliferation. Addition of Progestin causes down regulation of receptors. It also induces the enzyme $17 \beta$-Estradiol dehydrogenase, which transforms Estradiol (E2) to Estrone (E3). E3 has inferior activity. So, the histology of the endometrium changes from proliferative to secretory [9].

\section{Definition of Menopause}

The criteria for menopause laid down by National Comprehensive Cancer Network (NCCN); include a history of prior bilateral oophorectomy, age 60 years and above. Those below 60 years of age and amenorrhoeic for twelve months or longer in the absence of chemotherapy, tamoxifen or ovarian suppression, should have Estradiol (E2) and Follicle Stimulating Hormone (FSH) levels in the post menopausal range. Women on tamoxifen and below 60 years of age should have E2 and FSH levels in the post-menopausal range [10].

Premature menopause refers to menopause occurring more than two Standard Deviation (SD) below the mean average age for the population, although the age of 40 years is often used as a cut off. Premenopausal women have low FSH levels $(<10 \mathrm{mIU} / \mathrm{mL})$. In ovarian failure, either physiological or therapy induced, E2 levels decline and FSH levels increase. Menopausal FSH levels are usually $>40 \mathrm{mIU} / \mathrm{mL}$ [11].

\section{Treatments for Cancer Which May Result in Premature Menopause and/or Sexual Dysfunction}

1) Surgery and radiotherapy to the pelvis: for gynecological cancers [1]. 
2) Chemotherapy: it depends upon drugs used, cumulative dose and patient's age. Incidence of amenorrhea in survivors of breast cancer, treated with various regimen is as follows; $61 \%$ in patients aged < 40 years, and $95 \%$ in those aged $\geq 40$ years of age, in those treated with CMF (Cyclophosphamide, Methotrexate, 5 Fluorouracil). For FAC (5 Fluorouracil, Doxorubicin, Cyclophosphamide), AC (Doxorubicin, Cyclophosphamide), FEC (5 Fluorouracil, epirubicin, Cyclophosphamide), TAC (Docetaxel, Doxorubicin, Cyclophosphamide), it is 32.8\%, 34\%, 51\%, 51.4\% respectively [12]-[16].

3) Anti-estrogen therapy: the effects of anti-estrogen therapy mimic menopause and also contribute to ovarian dysfunction [15] [17].

4) Survivors with a history of Hematopoietic Stem Cell Transplant (HSCT): They have multiple types of sexual dysfunction even after 5 to 10 years. It may be related to Graft versus Host Disease (GvHD), such as vaginal fibrosis, stenosis, mucosal changes and vaginal bleeding. High dose of steroids lead to emotional lability. They also affect feelings of attractiveness and sexual activity [18].

Several mechanisms of chemotherapy induced early menopause have been described. In vitro studies suggest that chemotherapy leads to direct follicular damage due to apoptotic changes in pre-granulosa cells [19]. There is destruction of primordial follicles and impairment of follicular maturation. Ovarian cytotoxicity is irreversible, as women are born with a fixed number of germ cells, and these are not replenished [20].

\section{Various Studies on the Role of HRT in Menopause}

Two landmark studies which described the risks and benefits of HRT in healthy women were Million Women Study (MWS) and Women's Health Initiative Study (WHI). As per the WHI study, EPT lead to an increase in incidence of breast cancer and reduction in colorectal cancer.

There was no difference in the incidence of endometrial cancer. Use of ERT revealed no difference in the incidence of breast and colorectal cancers. The MWS had three arms. The EPT arm had an increased incidence of breast and ovarian cancer, while there was no difference in endometrial cancer. On the hand, the ERT arm and Tibolone arm, showed an increase in incidence of both, breast and endometrial cancers [1].

\subsection{Role of HRT in Survivors of Breast Cancer}

The annual incidence of breast cancer in the age group of 25 to 39 years is 91.3 per 100,000 lakh women [21]. Two Randomized Controlled Trials (RCTs), HABITS (HRT After Breast Cancer-Is iT Safe?) and Stockholm study, comparing menopausal HRT with no therapy in women with early stage breast cancer, were carried out in Sweden, in 1997 [1].

The HABITS Trial aimed to evaluate the potential risk of recurrent breast cancer in women using HRT. It was designed to enroll at least 1300 women and follow them up for five years. It was terminated early on December $17^{\text {th }}$, 2003, after a median follow-up of slightly longer than two years. This was because recurrent or de novo breast cancer had developed in 26 women in the HRT group and 7 women in the no-HRT group. There was an unacceptably high risk of breast cancer in breast cancer survivors allocated to receive HRT for menopausal symptoms. An unplanned subset analysis of the HABITS trial showed no increase in ER negative breast cancer on HRT [22].

Table 1 summarizes the risks and benefits of HRT in breast cancer survivors [22]-[24].

\subsection{Tibolone in Breast Cancer Survivors}

Tibolone acts on neuroendocrine circuitries, relieving hot flushes and mood swings. It acts on vaginal and clitoral tissues, causing vascular congestion and lubrication. LIBERATE (Livial Intervention following Breast cancer Efficacy, Recurrence and Tolerability Endpoints) was a randomized, placebo controlled, double-blind study, carried out from July 2002 to December 2004. It was stopped six months in advance. The primary endpoint was breast cancer recurrence. The secondary endpoints were vasomotor symptoms, bone mineral density (BMD), health-related quality of life and overall survival. 3148 women from 245 centres in 31 countries, treated for breast cancer, who had attained menopause, were enrolled in this study. At a median follow-up of 3.1 years, an increased risk of recurrence with the Tibolone users (HR 1.40) was observed. The risk of breast cancer recurrence was more evident in women with ER-positive tumors status than in ER-negative tumors [25].

Therefore, Estrogen replacement therapy is not recommended in women with a history of breast cancer, 
Table 1. Risks and benefits of HRT in breast cancer survivors [22]-[24].

\section{Risks of HRT}

Benefits of HRT

\section{Recurrence of breast cancer:}

HABITS trial: At a median follow up of 2.1 years, 26 patients in the HRT group and 7 patients in the non-HRT group had a new breast cancer event.

WHI trial: Women in the EPT group experienced a $26 \%$ increase in breast cancer (38 vs 30 per 10,000 person-years).

Lung cancer:

WHI trial: At a mean follow-up of 5.6 years and 2.4 years of additional follow-up, there was a trend towards increased lung cancer along with increased mortality. The risk continued even after cessation of HRT. This is of concern for smokers and for those who have other risk factors for lung cancer
Reduction in risk of fractures (WHI trial)

Possible decreased risk of colorectal cancer (WHI trial)

\section{Others:}

WHI trial: Women in the EPT group had an increased risk of coronary artery disease, stroke and pulmonary embolism.

including those who have hormone receptor negative disease, because of the increased risk of breast cancer recurrence [26].

\subsection{Role of HRT in Survivors of Epithelial Ovarian Cancer (EOC)}

Cytoreductive surgery leads to loss of ovarian function. Use of HRT is not recommended in Endometrioid epithelial ovarian cancer because of the potential stimulation of residual cancer cells and induction of new hormone-dependent disease. It may be used in other types of EOC [8].

\subsection{Role of HRT in Ovarian Germ Cell Tumor Survivors}

Fertility sparing surgery is possible in most cases. Adjuvant chemotherapy with Bleomycin, Etoposide, and Cisplatin results in gonadal dysfunction, leading to transient or permanent ovarian failure. These patients might benefit from HRT [8].

\subsection{Role of HRT in Survivors of Sex Cord Stromal Tumors}

The most common histopathological subtype is Granulosa cell tumor. About 30\% of these tumors are Estrogen Receptor (ER) positive and 100\% are Progesterone Receptor (PR) positive. Hence, HRT should not be used because of hormone dependent nature [8].

\subsection{Role of HRT in Endometrial Cancer Survivors}

$20 \%-25 \%$ of women with endometrial cancer are premenopausal. There are two types of endometrial cancer. Type I is Endometrioid, ER and PR positive, low grade and associated with prolonged use of estrogen and obesity. Type II is serous papillary or clear cell type, ER and PR negative, high grade and aggressive in nature [8]. A trial conducted by the Gynecologic Oncology Group (GOG) randomized women with Stage 1 and Stage 2 endometrial cancer after hysterectomy, to 3 years of conjugated estrogen, $0.625 \mathrm{mg}$ or placebo, but failed to recruit and was terminated early, when the WHI-E study was published. A preliminary report of 1236 women, at a median follow up of 35.7 months, demonstrated a recurrence rate of $2.3 \%$ in the HRT group vs. $1.6 \%$ in the placebo group. Deaths due to endometrial cancer were $0.8 \%$ in the HRT group and $0.6 \%$ in the placebo group. HRT is not recommended in survivors of endometrial cancer [27].

\subsection{Role of HRT in Survivors of Uterine Sarcoma}

Uterine sarcomas are rare tumors 93\% - 8\% of all uterine malignancies in women), hence data is limited. They include Leiomyosarcoma, endometrial stromal sarcoma, undifferentiated stromal sarcoma and carcinosarcoma. Endometrial stromal sarcomas are hormone sensitive. Estrogen therapy is contraindicated [8]. In a retrospective series of 22 patients, recurrence rates were $80 \%$ (4 out of 5 patients) in those who received HRT and 35\% (6 out 
of 17 patients) for those who did not [28]. Leiomyosarcoma is also a hormone sensitive tumor; it expresses estrogen and progesterone receptors. So, avoidance of estrogen therapy is recommended [8].

\subsection{Role of HRT in Survivors of Cervical Cancer}

External Beam Radiotherapy (EBRT) and Intra Cavitary Brachytherapy (ICBT) are indicated in advance stages. This leads to iatrogenic menopause, vaginal Stenosis and dyspareunia. There is no clear evidence about a linkage between hormonal therapy and adverse outcome in cervical cancer. HRT is not contraindicated in survivors of cervical squamous cell cancer. However, caution must be exercised in cervical adenocarcinoma, as this subtype is estrogen dependent [8].

\subsection{Survivors of Other Malignancies}

HRT is not a risk factor for the development of non-endocrine dependent malignancies. There is no RCT to show that HRT use is detrimental in this group. In those rendered menopausal following treatment of leukemia and lymphoma, HRT may be prescribed upto 51 years of age [1].

\subsection{Role of HRT in Osteoporosis}

Estrogen has anti-resorptive properties with proven anti-fracture efficacy. This was demonstrated in the WHI study. Estrogen therapy alone and combined estrogen and progesterone were associated with a $33 \%$ to $34 \%$ reduction in hip fracture. In women with chemotherapy induced menopause who are not at increased risk for breast cancer, replacement of estrogen/progesterone until the normal age of menopause is very likely to be beneficial for bone health [26].

However, as already mentioned earlier, estrogen replacement therapy is not recommended in women with a history of breast cancer [26].

\section{When Do We Commence HRT, at What Dose and for How Long?}

HRT is to be initiated after six months after completion of treatment (chemotherapy or radiation therapy) to reduce the thrombotic risk due to cancer and its treatment [8].

As per the updates 2013 International Menopause Society recommendations on menopausal hormone therapy, HRT must be individualized and tailored according to the symptoms, personal history (age, time since menopause, risk of venous thromboembolism, stroke, ischemic heart disease and breast cancer), preferences and expectations of the woman. It is recommended until the average age of natural menopause. Data from the WHI study support safe use for at least five years in healthy women starting treatment before 60 years of age. Continued use may be appropriate based on individual risk profile [29].

Lower doses of HRT can reduce symptoms and maintain quality of life in many women. However, long term data regarding risks of fracture, cancer and cardiovascular complications are lacking [29]. Androgen replacement should be considered for women with diminished sexual desire and arousal.

\section{Newer Agents in the Management of Symptoms of Menopause}

A phase I clinical study was conducted using ultra-low-dose Estrone (E3) and Lactobacillus acidophilus vaginal tablets, for vaginal atrophy in post-menopausal breast cancer patients on aromatase inhibitors (AIs). AIs worsen or induce vaginal atrophy, dryness and dyspareunia. The most effective therapy is vaginal estrogen application. Vaginal administration of any dose of Estradiol (E2), increases serum levels of estrogen, which in turn can stimulate growth of breast cancer cells. Hence, E3, which is less potent,was preferred. It did not increase serum estrogen levels, or endometrial proliferation. Each tablet consists of 100 million viable L. acidophilus KS400 and $0.03 \mathrm{mg}$ E3. One tablet was inserted deep into the vagina before sleep for four weeks, followed by maintenance therapy with three vaginal tablets weekly, for eight weeks. The benefits of E3 are that it cannot be transformed back to E2. It has a sorter nuclear retention time, of less than 6 hours. It has low affinity to the nuclear receptor and plasma proteins and has rapid metabolic clearance. Continuous and high doses of E3 are not achieved. This is because, vaginal absorption of E3 decreases as the vaginal epithelium matures over a few days to weeks. Similar studies could be conducted in young women with menopausal symptoms of cancer [30]. 
Table 2. Role of HRT in survivors of various malignancies.

\begin{tabular}{|c|c|c|}
\hline Site & Histopathological type & Is HRT indicated? \\
\hline Breast & Any & No \\
\hline \multirow[t]{4}{*}{ Ovary } & $\begin{array}{l}\text { Endometriod type of epithelial } \\
\text { ovarian cancer }\end{array}$ & No \\
\hline & Others & Yes \\
\hline & Germ cell tumor & Yes \\
\hline & Sex cord stromal tumor & No \\
\hline \multirow[t]{3}{*}{ Uterus } & Type 1 endometrial cancer & No \\
\hline & Type 2 endometrial cancer & No \\
\hline & $\begin{array}{l}\text { Endometrial stromal sarcoma } \\
\text { Leiomyosarcoma }\end{array}$ & No \\
\hline \multirow[t]{3}{*}{ Cervix } & Adenocarcinoma & No \\
\hline & Squamous cell carcinoma & Yes \\
\hline & Others: & \\
\hline \multicolumn{2}{|c|}{$\begin{array}{l}\text { Leukemia and lymphoma, survivors with a history of } \\
\text { hematopoietic stem cell transplant }\end{array}$} & Yes \\
\hline \multicolumn{2}{|c|}{ Other non-endocrine dependent malignancies } & Yes \\
\hline
\end{tabular}

Ospemifene is a novel Selective Estrogen Response Modifier (SERM). It is indicated in post menopausal women with moderate to severe dyspareunia due to vulvar and vaginal atrophy, without known or suspected breast cancer and without a history of breast cancer. It is a $60 \mathrm{mg}$ tablet, administered orally, once daily. It is recommended by NCCN for dyspareunia in survivors, but only if the cancer was not hormone sensitive [31].

\section{Conclusion}

HRT is contraindicated in survivors of breast cancer, endometrioid type of EOC, sex cord stromal tumors, endometrial cancer, leiomyosarcoma and endometrial stromal sarcoma of the uterus. Caution needs to be exercised in adenocarcinoma of the cervix. It is not contraindicated in survivors of other types of EOC, germ cell tumor and squamous cell carcinoma of the cervix (Table 2).

Young women who have survived the onslaught of cancer and its treatment are also faced with additional emotional and physical hurdles. They may attain menopause several years earlier than expected. This will have a negative impact on their quality of life. While several non-hormonal therapies are available to combat the symptoms of premature menopause, development of less potent and yet effective hormonal therapies are the need of the hour.

\section{Conflicts of Interest}

There are no conflicts of interest to be declared.

\section{References}

[1] King, J., Wynne, C.H., Assersohn, L. and Jones, A. (2011) Hormone Replacement Therapy and Women with Premature Menopause-A Cancer Survivorship Issue. European Journal of Cancer, 47, 1623-1632. http://dx.doi.org/10.1016/j.ejca.2011.04.007

[2] Schover, L.R. (2008) Premature Ovarian Failure and Its Consequences: Vasomotor Symptoms, Sexuality, and Fertility. Journal of Clinical Oncology, 26, 753-758. http://dx.doi.org/10.1200/JCO.2007.14.1655

[3] Cusack, L., Brennan, M., Baber, R. and Boyle, F. (2013) Menopausal Symptoms in Breast Cancer: Management Update. British Journal of General Practice, 63, 51-52. http://dx.doi.org/10.3399/bjgp13X660977 
[4] Finck, G., Barton, D.L., Loprinzi, C.L., et al. (1998) Definitions of Hot Flashes in Breast Cancer Survivors. Journal of Pain and Symptom Management, 16, 327-333. http://dx.doi.org/10.1016/S0885-3924(98)00090-6

[5] Kronenberg, F. (1994) Hot Flashes: Phenomenology, Quality of Life, and Search for Treatment Options. Experimental Gerontology, 29, 319-336. http://dx.doi.org/10.1016/0531-5565(94)90012-4

[6] Ganz, P.A., Greendale, G.A., Petersen, L., et al. (2003) Breast Cancer in Younger Women: Reproductive and Late Health Effects of Treatment. Journal of Clinical Oncology, 21, 4184-4193. http://dx.doi.org/10.1200/JCO.2003.04.196

[7] Hickey, M., Saunders, C.M. and Stuckey, B.G.A. (2005) Management of Menopausal Symptoms in Patients with Breast Cancer: An Evidence-Based Approach. The Lancet Oncology, 6, 687-695. http://dx.doi.org/10.1016/S1470-2045(05)70316-8

[8] Perrone, A.M., Pozzati, F., Santini, D., Rossi, M., Procaccini, M., Casalini, L., et al. (2014) Gynecological Malignancies and Hormonal Therapies: Clinical Management and Recommendations. World Journal of Obstetrics and Gynecology, 3, 162-170.

[9] Casper, R.F. (1996) Regulation of Estrogen/Progestogen Receptors in the Endometrium. International Journal of Fertility and Menopausal Studies, 41, 16-21.

[10] NCCN Guidelines Version 2. 2013 Invasive Breast Cancer, Definition of Menopause. http://www.nccn.org/

[11] Murthy, V. and Chamberlain, R.S. (2012) Menopausal Symptoms in Young Survivors of Breast Cancer-A Growing Problem without an Ideal Solution. Cancer Control, 19, 317-329.

[12] Minton, S.E. and Munster, P.N. (2002) Chemotherapy-Induced Amenorrhea and Fertility in Women Undergoing Adjuvant Treatment for Breast Cancer. Cancer Control, 9, 466-472.

[13] Anderson, R.A., Themmen, A.P., Al-Qahtani, A., Groome, N.P. and Cameron, D.A. (2006) The Effects of Chemotherapy and Long-Term Gonadotrophin Suppression on the Ovarian Reserve in Premenopausal Women with Breast Cancer. Health Human Reproduction, 21, 2583-2592. http://dx.doi.org/10.1093/humrep/del201

[14] Tominaga, T., Morimoto, T. and Ohasi, Y., for the Japan Letrozole Study Group (2000) A Pivotal Double-Blind Trial in Japan of an Aromatase Inhibitor Letrozole (Third Generation) vs. Its Predecessor Fadrozole Hydrochloride (Second Generation). Annals of Oncology, 11, 25.

[15] Tham, Y.L., Sexton, K., Weiss, H., Elledge, R., Friedman, L.C. and Kramer, R. (2007) The Rates of Chemotherapy-Induced Amenorrhea in Patients Treated with Adjuvant Doxorubicin and Cyclophosphamide Followed by Ataxane. American Journal of Clinical Oncology, 30, 126-132. http://dx.doi.org/10.1097/01.coc.0000251398.57630.4f

[16] Lutchman, S.K., Muttukrishna, S., Stein, R.C., McGarrigle, H.H., Patel, A., Parikh, B., et al. (2007) Predictors of Ovarian Reserve in Young Women with Breast Cancer. British Journal of Cancer, 96, 1808-1816. http://dx.doi.org/10.1038/sj.bjc.6603814

[17] Oktay, K., Oktem, O., Reh, A. and Vahdat, L. (2006) Measuring the Impact of Chemotherapy on Fertility in Women with Breast Cancer. Journal of Clinical Oncology, 24, 4044-4046. http://dx.doi.org/10.1200/JCO.2006.06.9823

[18] Thygesen, K.H., Schjødt, I. and Jarden, M. (2012) The Impact of Hematopoietic Stem Cell Transplantation on Sexuality: A Systematic Review of the Literature. Bone Marrow Transplantation, 47, 716-724. http://dx.doi.org/10.1038/bmt.2011.169

[19] Meirow, D. (2000) Reproduction Post-Chemotherapy in Young Cancer Patients. Molecular and Cellular Endocrinology, 169, 123-131. http://dx.doi.org/10.1016/S0303-7207(00)00365-8

[20] Blumenfeld, Z. (2002) Preservation of Fertility and Ovarian Function and Minimalization of Chemotherapy Associated Gonadotoxicity and Premature Ovarian Failure: The Role of Inhibin-A and -B as Markers. Molecular and Cellular Endocrinology, 187, 93-105. http://dx.doi.org/10.1016/S0303-7207(01)00712-2

[21] Pagani, O. and Goldhirsch, A. (2006) Breast Cancer in Young Women: Climbing for Progress in Care and Knowledge. Women's Health, 2, 717-732. http://dx.doi.org/10.2217/17455057.2.5.717

[22] Holmberg, L. and Anderson, H. (2004) HABITS Steering and Data Monitoring Committees. HABITS (Hormonal Replacement Therapy after Breast Cancer-Is It Safe?), a Randomized Comparison: Trial Stopped. Lancet, 363, 453-455. http://dx.doi.org/10.1016/S0140-6736(04)15493-7

[23] Rossouw, J.E., Anderson, G.L., Prentice, R.L., et al. (2002) Risks and Benefits of Estrogenplus Progestin in Healthy Postmenopausal Women: Principal Results from the Women's Health Initiative Randomized Controlled Trial. JAMA, 288, 321-333. http://dx.doi.org/10.1001/jama.288.3.321

[24] Chlebowski, R.T., Schwartz, A.G., Wakelee, H., et al. (2009) Oestrogen plus Progestinand Lung Cancer in Postmenopausal Women (Women's Health Initiative Trial): Apost-Hoc Analysis of a Randomised Controlled Trial. Lancet, 374, 1243-1251. http://dx.doi.org/10.1016/S0140-6736(09)61526-9

[25] Kenemans, P., Bundred, N.J., Foidart, J.M., Kubista, E., von Schoultz, B., Sismondi, P., et al. (2009) Safety and Efficacy of Tibolone in Breast-Cancer Patients with Vasomotor Symptoms: A Double-Blind, Randomized, Non-Inferiority 
Trial. Lancet Oncology, 10, 135-146. http://dx.doi.org/10.1016/S1470-2045(08)70341-3

[26] Gralow, J.R., Blermann, J.S., Farooki, A., Fornier, M.N., Gagel, R.F., Kumar, R., et al. (2013) NCCN Task Force Report: Bone Health in Cancer Care. Journal of the National Comprehensive Cancer Network: JNCCN, 11, S1-S50.

[27] Barakat, R.R., Bundy, B.N., Spirtos, N.M., Bell, J. and Mannel, R.S. (2006) Randomized Double-Blind Trial of Estrogen Replacement Therapy versus Placebo in Stage I or II Endometrial Cancer: A Gynecologic Oncology Group Study. Journal of Clinical Oncology, 24, 587-592. http://dx.doi.org/10.1200/JCO.2005.02.8464

[28] Chu, M.C., Mor, G., Lim, C., Zheng, W., Parkash, V. and Schwartz, P.E. (2003) Low-Grade Endometrial Stromal Sarcoma: Hormonal Aspects. Gynecologic Oncology, 90, 170-176. http://dx.doi.org/10.1016/S0090-8258(03)00258-0

[29] de Villiers, T.J., Pines, A., Panay, N., Gambacciani, M., Archer, D.F., Baber, R.J., et al., on Behalf of the International Menopause Society (2013) Updated 2013 International Menopause Society Recommendations on Menopausal Hormone Therapy and Preventive Strategies for Midlife Health. Climacteric, 16, 316-337. http://dx.doi.org/10.3109/13697137.2013.795683

[30] Donders, G., Neven, P., Moegele, M., Lintermans, A., Bellen, G., Prasauskas, V., et al. (2014) Ultra-Low-Dose Estriol and Lactobacillus acidophilus Vaginal Tablets (Gynoflor ${ }^{\circledR}$ ) for Vaginal Atrophy in Postmenopausal Breast Cancer Patients on Aromatase Inhibitors: Pharmacokinetic, Safety, and Efficacy Phase I Clinical Study. Breast Cancer Research and Treatment, 145, 371-379. http://dx.doi.org/10.1007/s10549-014-2930-X

[31] NCCN Guidelines Version 2. 2015, Survivorship, Discussion, MS-30. http://www.nccn.org/

\section{Abbreviations Used in This Article}

CAMS: Cancer and menopause study

E2: Estradiol

E3: Estrone

EBRT: External Beam Radiotherapy

EOC: Epithelial ovarian cancer

EOT: Estrogen replacement therapy

EPT: Estrogen-plus-progesterone therapy

ER: Estrogen receptor

FSH: Follicle Stimulating Hormone

GOG: Gynecologic Oncology Group

GvHD: Graft versus host disease

HABITS trial: HRT After Breast Cancer-Is It Safe?

HRT: Hormone Replacement Therapy

HSCT: Hematopoietic Stem Cell Transplant

ICBT: Intra Cavitary Brachytherapy

LIBERATE trial: Livial Intervention following Breast cancer Efficacy, Recurrence and Tolerability Endpoints trial MWS: Million Women Study

NCCN: National Comprehensive Cancer Network

PR: Progesterone receptor

RCT: Randomized Control Trial

SD: Standard deviation

WHI trial: Women’s Health Initiative trial 